\title{
Industrial Group Production Program under market uncertainty *
}

\author{
Dorota Bochnacka * \\ * Faculty of Management and Computer Modelling \\ Kielce University of Technology Poland, 25-314 Kielce, Poland \\ (e-mail: dbochnacka@tu.kielce.pl).
}

Keywords: optimal control, industrial group

\section{INTRODUCTION}

The effects of globalization, such as the development of economies, free movement of capital, whether the increase in competitiveness is forcing companies to adjust strategy to rapidly changing environmental conditions. One of the ways to achieve market success and maintain a competitive position is cooperating with others enterprises.

In this work, we are concentrated on an industrial group with vertical cooperation in which, legally and economically independent companies of different stages of the technological chain are working together in the direction of production cost reduction, setting transfer price for goods and services, and impact on the final consumer (see Fernandez-Olmos et al. (2016)).

The goal of the work is to define of those conditions of cooperation of the group members which could ensure their stable functioning and economic development and could maximize economic efficiency on the long-term planning horizon under uncertain behavior of consumers.

\section{OPTIMAL COOPERATION PROBLEM}

Let us consider some industrial group on a cooperation interval $\left[t_{0}, t_{1}\right]$, where $t_{0}$ and $t_{1}$ are the initial and final moments of cooperation $\left(t_{0} \leq t_{1},\left[t_{0}, t_{1}\right] \subset \mathbb{R}_{+}\right)$. Let $j \in\{1,2, \ldots, K\}$. This structure includes the vertical cooperation of $k$ members. Each member acquires the necessary resources and has production facilities. The manufacturing activity of the group is considered on product and consumer markets. We assume that the production does not bring the loss to $j^{\text {th }}$ member of the group, $j \in\{1,2, \ldots, K\}$, when its selling price covers the manufacturing costs as well as that the overall production does not exaggerate the market demand.

Each member of the group tries to improve own economic situation and to increase profit. Analyzing the internal and external characteristics, we have noticed the following. There exist some parameters, for example, the products internal transfer prices, which present the antagonistic interests of the members of the group, and therefore these values have an effect not only on the profit of each group's member but also on the decision of cooperation and

^ This paper was supported by Ministry of Science and High Educa- economic efficiency on the planning horizon. Moreover, the uncertainty of market characteristics constrains the economic activities of the group as well as of each its member.

The problem of the maximizing of economic efficiency on long-term planning horizon $\left[t_{0}, t_{1}\right]$ can be formulated as the task of the functional maximization with respect to control variables $u(t)\left(x_{j} \in \varepsilon, u \in U, \varepsilon\right.$ and $U$ are some spaces)

$$
\pi_{j}\left(t_{1}\right)=\max _{\mathbf{u} \in U} \sum_{j=1}^{K}\left[J_{j}\left(x_{j}(\cdot), u(\cdot)\right)\right]
$$

s.t.

$$
\begin{gathered}
x_{j}(t)=\mathbb{T}\left(t, x_{j}(t), u(t)\right) \\
\Psi_{j}^{1}\left(x_{j}\left(t_{0}\right), x_{j}\left(t_{1}\right)\right) \leq 0 \\
\Psi_{j}^{2}\left(x_{j}\left(t_{0}\right), x_{j}\left(t_{1}\right)\right)=0 \\
g_{j}^{k}\left(t, x_{j}(t)\right) \leq 0, k=1,2, \ldots, \ell_{1}^{(j)} \\
\phi_{j}^{m}(t, u(t)) \leq 0, m=1,2, \ldots, \ell_{2}^{(j)}
\end{gathered}
$$

where $\mathbb{T}\left(t, x_{j}(t), u(t)\right)$ is a differential operator of the mathematical model describing the economic situation $x_{j}(t) \in \mathbb{R}$ of $j^{t h}$ member of group, $\Psi_{j}^{1}: \mathbb{R} \times \mathbb{R} \rightarrow \mathbb{R}^{n_{1}^{(j)}}$ and $\Psi_{j}^{2}: \mathbb{R} \times \mathbb{R} \rightarrow \mathbb{R}_{2}^{n_{2}^{(j)}}$ are the terminal constraints; $g_{k}^{(j)}: \mathbb{R} \times$ $\mathbb{R} \rightarrow \mathbb{R}_{1}^{\ell_{1}^{(j)}}$ are phase constraints; $\phi_{m}^{(j)}: \mathbb{R} \times \mathbb{R}^{r} \rightarrow \mathbb{R}^{\ell_{2}^{(j)}}$ are the control constraints; $n_{1}^{(j)}, n_{2}^{(j)}, \ell_{1}^{(j)}, \ell_{2}^{(j)}, r \in \mathbb{N}$.

The solution of the optimal control problem (1) - (6) depends on the model selection for each its expression. Bochnacka and Filatova (2017) studied the case of the twoenterprises group. The stochastic differential equation with fractional Brownian motion was used as the mathematical model describing the economic situation $x_{j}(t)$. Taking into account the theoretical results proposed by Filatova et al. (2010) the necessary optimality conditions were formulated. Since the real economic situation is more complex, the incorporation of the market uncertainty would allow to check the reaction (a kind of sensitivity) of the economic situation. For this purpose, we propose to take into account the consumers behavior.

\section{MODEL OF CONSUMER BEHAVIOR}

Having in mind the external influence of the product market, it is assumed that the value of total sales of the 
group during given period $\left[t_{0}, t_{1}\right]$ depends on demand and, by implication, on quantity of consumers willing to buy products, on their behavior and decision.

Filatova et al. (2016) proposed the model of consumer behavior, which can be implemented through the composition of individual choices over two alternative policies Rawlsian maximin and Harsanyian utilitarianism. Analysis of similar conceptual and mathematical models describing the social preferences pointed out the possibilities of the consumer behavior dynamics' description omitting any specific analysis of decision-making mechanisms.

Here we suppose that the number of consumers willing to buy the products of the group at some moment of time $t \in\left[t_{0}, t_{1}\right]$ is $y(t)>0, y(t) \in \mathbb{R}_{+}$. The consumers are subdivided into different classes according to the policy of its members. Each consumer can choose (and change or stay with his/her decision later) a situation that favors the less well-off possibly in detriment of the most well-off (first group, "maximin"), or wants to maximize the average distribution of wealth (second group, mean utilitarianism or "maximean"), or finally desires to maximize most welloff, possibly to the detriment of the less well-off, especially if it maximizes the general well-off (third group, which can label "maximax" and corresponds to some classical form of unqualified utilitarianism). In this case, consumers can be defined through:

$$
y(t)=y^{(1)}(t)+y^{(2)}(t)+y^{(3)}(t)
$$

where $y^{(\ell)}(t) \geq 0, y^{(\ell)}(t) \in \mathbb{R}_{+}$, represents the cardinality of $\ell^{\text {th }}$ group, $\bar{\ell}=1,2,3$.

As one can see, there exist six possibilities for change. Let the members of each group change their strategies with six different intensities. These intensities depend on social preference, risk aversion or other exogenous factors such as culture, etc.). We denote these parameters as $\gamma_{i j}$, which corresponds to exchange between $i$ and $j, i, j=1,2,3$. The passage of individuals from the group to group usually occurs as a result of interpersonal contacts among individuals or other factors which make an individual change the preferences. This phenomenon cannot be measured directly.

Taking into consideration the characteristics of the consumers mentioned above, the dynamic model of consumer behavior can be written as the system of ordinary differential equations:

$$
\begin{aligned}
d y^{(1)}(t)= & {\left[-\left(\gamma_{21}-\gamma_{12}\right) y^{(1)}(t) y^{(2)}(t)\right.} \\
& \left.+\gamma_{23} y^{(3)}(t)-\gamma_{32} y^{(1)}\right] d t, \\
d y^{(2)}(t)= & {\left[\left(\gamma_{21}-\gamma_{12}\right) y^{(1)}(t) y^{(2)}(t)\right.} \\
& \left.-\left(\gamma_{32}-\gamma_{23}\right) y^{(2)}(t) y^{(3)}(t)\right] d t, \\
d y^{(3)}(t)= & {\left[\left(\gamma_{32}-\gamma_{23}\right) y^{(2)}(t) y^{(3)}(t)\right.} \\
& \left.-\gamma_{23} y^{(3)}(t)+\gamma_{32} y^{(1)}(t)\right] d t,
\end{aligned}
$$

with $y^{(\ell)}\left(t_{0}\right)=y_{0}^{(\ell)}$.

Associating with each class $y^{(\ell)}(t)$ some purchasing power $a_{j}^{(\ell)}, j=1,2, \ldots, K$, the demand on the production can be presented as some function

$$
d_{j}(t)=\xi\left(t, \sum_{\ell=1}^{3} a_{j}^{(\ell)} y^{(\ell)}(t)\right), j=1,2, \ldots, K .
$$

Under these assumptions the formulation of the problem of the maximizing of economic efficiency under conditions of dynamic demand on production is the same as in previous case, however one of groups of the phase constraints (5), which take a form

$$
x_{j}(t)-d_{j}(t) \leq 0 \forall t \in\left[t_{0}, t_{1}\right], j=1,2, \ldots, K .
$$

requires particular attention due to complex behavior near the equilibrium points of (8).

\section{CONCLUSIONS}

An industrial group, consisting of two enterprises with vertical cooperation, is considered. We assume that both enterprises, having necessary production capacities, act on the product markets once as the partners and once as the independent actors. The main problem of this kind of cooperation is to find the optimal conditions for the production programs for both enterprises, or in other words, it is necessary to find the solution of task (1) - (6) under different conditions of the consumer's behavior. The possible way of the mathematical problem formulation, as well as its relation to the optimal control problem, is the essence of this work. The theoretical backgrounds for the solution of the optimal cooperation production program are developed using the Dubovitski-Milyutin method.

In the further stages of the research, issues related to determining the determinants of vertical cooperation will be introduced, which should contribute to the formulation of more precise models of cooperating enterprises and their optimal management.

\section{REFERENCES}

Bochnacka, D. and Filatova, D. (2017). Necessary optimality conditions for enterprises production programs. In The International Conference of Information and Digital Technologies 2017, 61-65.

Fernandez-Olmos, M., Dejo-Oricain, N., and RosellMartinez, J. (2016). Product differentiation strategy and vertical integration: an application to the doc rioja wine industry. Journal of Business Economics and Management, 17(5), 796-809.

Filatova, D., Bourgeois-Gironde, S., Baratgin, J., Jamet, F., and Shao, J. (2016). Cycles of maximin and utilitarian policies under the veil of ignorance. Mind \& Society, 15(1), 105-116.

Filatova, D., Grzywaczewski, M., and Osmolovskii, N. (2010). Optimal control problem with an integral equation as the control object. Nonlinear Analysis: Theory, Methods and Applications, 72(3), 1235 - 1246. 